\title{
Pretubulysin: a new option for the treatment of metastatic cancer
}

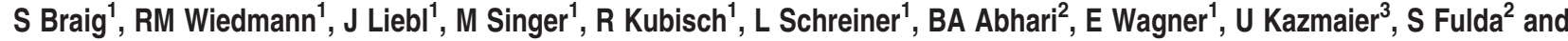 \\ AM Vollmar,
}

Tubulin-binding agents such as taxol, vincristine or vinblastine are well-established drugs in clinical treatment of metastatic cancer. However, because of their highly complex chemical structures, the synthesis and hence the supply issues are still quite challenging. Here we set on stage pretubulysin, a chemically accessible precursor of tubulysin that was identified as a potent microtubule-binding agent produced by myxobacteria. Although much simpler in chemical structure, pretubulysin abrogates proliferation and long-term survival as well as anchorage-independent growth, and also induces anoikis and apoptosis in invasive tumor cells equally potent to tubulysin. Moreover, pretubulysin posseses in vivo efficacy shown in a chicken chorioallantoic membrane (CAM) model with T24 bladder tumor cells, in a mouse xenograft model using MDA-MB-231 mammary cancer cells and finally in a model of lung metastasis induced by 4T1 mouse breast cancer cells. Pretubulysin induces cell death via the intrinsic apoptosis pathway by abrogating the expression of pivotal antiapoptotic proteins, namely Mcl-1 and Bcl- $\mathrm{x}_{\mathrm{L}}$, and shows distinct chemosensitizing properties in combination with TRAIL in two- and three-dimensional cell culture models. Unraveling the underlying signaling pathways provides novel information: pretubulysin induces proteasomal degradation of Mcl-1 by activation of mitogen-activated protein kinase (especially JNK (c-Jun N-terminal kinase)) and phosphorylation of Mcl-1, which is then targeted by the SCF ${ }^{\mathrm{Fbw7}} \mathrm{E} 3$ ubiquitin ligase complex for ubiquitination and degradation. In sum, we designate the microtubule-destabilizing compound pretubulysin as a highly promising novel agent for mono treatment and combinatory treatment of invasive cancer.

Cell Death and Disease (2014) 5, e1001; doi:10.1038/cddis.2013.510; published online 16 January 2014

Subject Category: Cancer

Cancer metastasis is a complex process where tumor cells migrate and invade the surrounding tissue, intravasate into the blood flow and extravasate into specific organs where they finally colonize. ${ }^{1}$ Besides being highly mobile and invasive, tumor cells have to escape apoptosis to survive in foreign environment during metastasis. ${ }^{2}$ In fact, apoptosis has been proposed as one determinant factor that modulates metastasis efficiency at crucial steps in the metastatic pathway such as the initial steps of detachment of tumor cells from the extracellular matrix where cells have to survive in the absence of normal matrix components, the period where solitary cells have to survive in the circulation by opposing mechanical stress and immunological attacks and finally in the phase after extravasation during micrometastases formation in another organ. ${ }^{3-5}$ Current chemotherapeutic therapies are often ineffective against metastasis. Thus, new therapeutic agents and treatment regimens addressing hallmarks of metastasis are greatly needed to improve the prognosis for this yet incurable stage of the disease. ${ }^{6}$
In anticancer drug discovery, natural compounds have a long and successful history as reviewed by Newman and Cragg. ${ }^{7,8}$ Natural compounds are a product of interest because of their enormous chemical diversity and biological activities appointing them as 'privileged structures'. An apparent obstacle of natural products however is their supply, production and isolation as well as their chemical synthesis that is very often rather complex. ${ }^{9-11}$ Microbial sources such as actinomycetes and more recently myxobacteria are recognized as highly prolific producers of bioactive secondary metabolites, which open up biotechnological approaches to guarantee validated supply of novel experimental drug candidates in the battle against cancer. $^{12}$ Epothilones produced by myxobacteria Sorangium cellulosum are the most prominent example, as ixabepilone, a semisynthetic derivate has recently been approved by the US Food and Drug Administration for treatment of metastatic breast cancer. $^{13}$

Tubulysins display another group of highly potent experimental anticancer drugs produced by other myxobacterial

\footnotetext{
${ }^{1}$ Department of Pharmacy, Center for Drug Research, Pharmaceutical Biology, University of Munich, Butenandtstrasse 5-13, 81377 Munich, Germany; ${ }^{2}$ Institute for Experimental Research in Pediatrics, University Hospital Frankfurt, Komturstrasse 3a, 60528 Frankfurt aM, Germany and ${ }^{3}$ Institute for Organic Chemistry, Saarland University, PO Box 151150, 66041 Saarbrücken, Germany

${ }^{*}$ Corresponding author: AM Vollmar, Department of Pharmacy, Center for Drug Research, Pharmaceutical Biology, University of Munich, Butenandtstrasse 5-13, 81377 Munich, Germany. Tel: + 49892180 77172; Fax: + 49892180 77170; E-mail: angelika.vollmar@ cup.uni-muenchen.de

Keywords: microtubule-targeting agents; natural compounds; metastatic cancer; Fbw7; Mcl-1; TRAIL

Abbreviations: CAM, chicken chorioallantoic membrane; JNK, c-Jun N-terminal kinase; MTT, 3-[4,5-dimethylthiazol-2-yl]-2,5 diphenyl tetrazolium bromide; PARP, poly(ADP-ribose)-polymerase 1; PT, pretubulysin; SCID, severe-combined immunodeficiency; TRAIL, TNF-related apoptosis-inducing ligand; Tub A, tubulysin A; TUNEL, TdT-mediated dUTP-biotin nick-end labeling

Received 15.10.13; revised 19.11.13; accepted 20.11.13; Edited by A Stephanou
} 
strains such as Archangium gephyra and Angiococcus discoformis. Unfortunately, tubulysins are only obtained in limited amounts as fermentation, isolation procedures as well as intricate chemical synthesis are quite challenging. ${ }^{14-16}$ Pretubulysin, a natural precursor of tubulysins, although also of minute quantity in myxobacteria, was successfully synthesized in a large-scale setup ${ }^{17}$ and firstly tested for its bioactivity and target in some tumor cell lines. ${ }^{18-20}$

Here we propose pretubulysin as a potent and innovative experimental drug for metastatic cancer treatment, as pretubulysin not only induces apoptotic cell death in various cancer cell lines at nanomolar concentrations but also inhibits metastasis and tumor growth in vitro and in vivo. Pretubulysin is shown to augment the protein degradation of the antiapoptotic player $\mathrm{Mcl}-1$ by affecting the ubiquitin ligase SCF with its main component Fbw7 via the JNK (c-Jun $\mathrm{N}$-terminal kinase) pathway. In addition, pretubulysinmediated downregulation of $\mathrm{Mcl}-1$ provides the mechanistic base for a synergistic interaction with TRAIL (TNF-related apoptosis-inducing ligand), another candidate for adjuvant therapies for metastatic cancer. ${ }^{21}$

\section{Results}

Pretubulysin disrupts the microtubule network at nanomolar concentrations and leads to a G2/M arrest. Similar to the already characterized tubulysin A, pretubulysin, which represents a biosynthetic precursor compound of the tubulysins, disrupted the microtubule network at $10 \mathrm{nM}$ in T24 bladder carcinoma cells after $24 \mathrm{~h}$ as well as in MDA-MB-231 breast cancer cells after $16 \mathrm{~h}$ (Figure 1a). In line, analysis of the cell cycle progression revealed that treatment with both compounds for $48 \mathrm{~h}$ leads to a strong G2/M arrest (Figure 1b), as measured by FACS analysis.

Pretubulysin inhibits tumor cell growth. Classical proliferation assays that run for $72 \mathrm{~h}$ revealed that treatment of the highly aggressive and metastatic cancer cell lines T24 and MDA-MB-231 with pretubulysin at low nanomolar concentrations results in inhibition of proliferation, which is comparable to the effect of tubulysin $A\left(\mathrm{IC}_{50}\right.$ values: pretubulysin $-1.6 \mathrm{nM}$ in T24 cells and $2.4 \mathrm{nM}$ in MDA-MB-231 cells; tubulysin A $0.6 \mathrm{nM}$ in $\mathrm{T} 24$ and $1.9 \mathrm{nM}$ in MDA-MB-231 cancer cells,
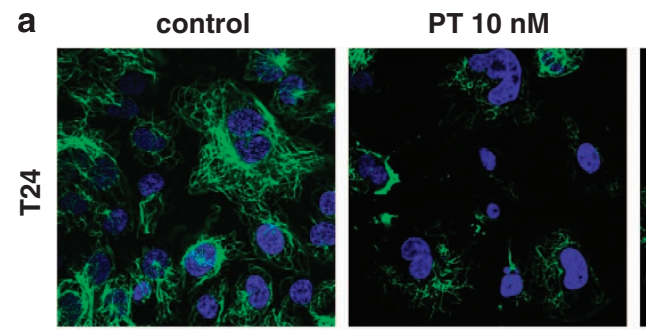

Tub A $10 \mathrm{nM}$
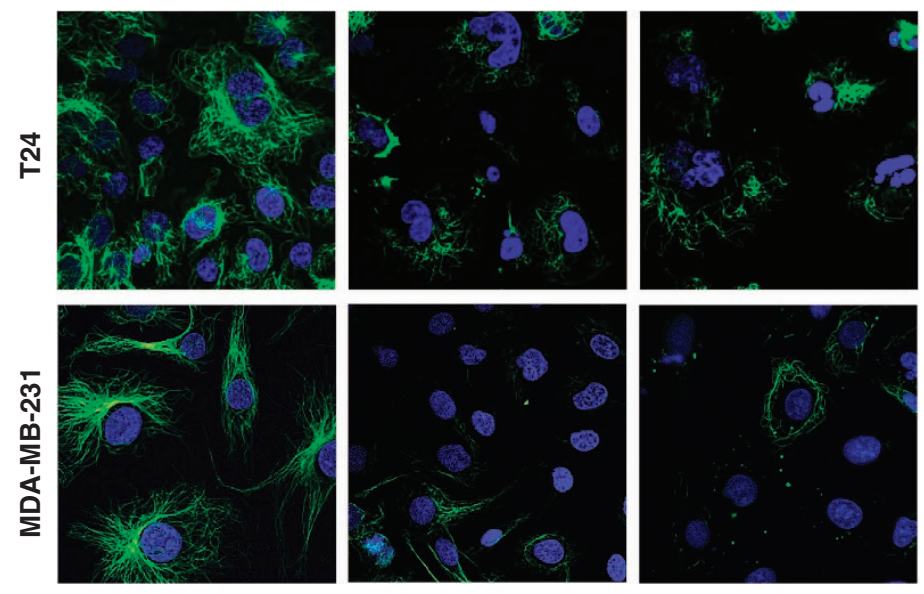

microtubule

nuclei

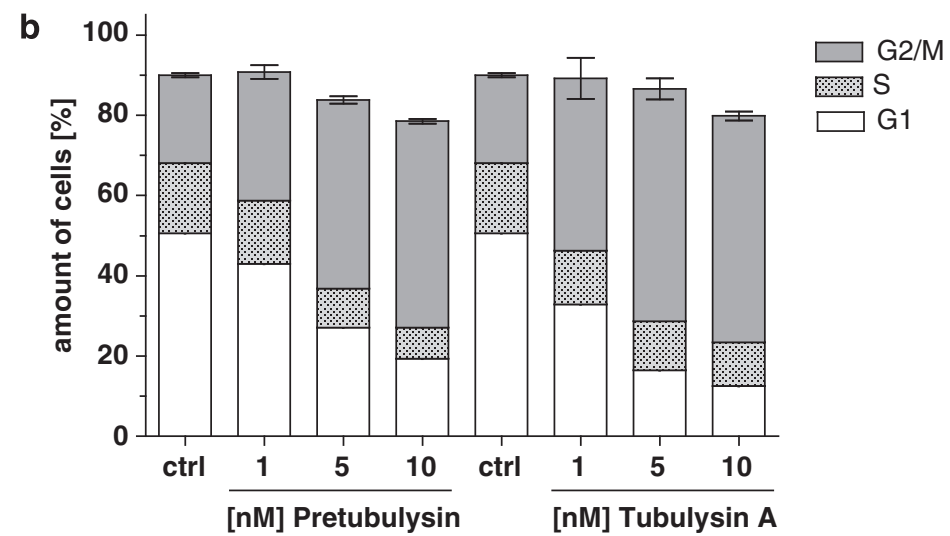

Figure 1 Pretubulysin (PT) and tubulysin A (Tub A) depolymerize microtubules. (a) T24 and MDA-MB-231 cells were treated for 24 or 16 h, respectively, with PT or Tub A as indicated. After fixing and permeabilization, tubulin fragments were washed out as described in Materials and Methods. Staining with anti- $\alpha$-tubulin antibody and Hoechst 33258 demonstrated a strong disruption of the microtubule network. Representative images out of three independent experiments are shown. (b) Treatment of MDA-MB-231 cells with PT or Tub A results in G2/M arrest, as shown by fluorescence-activated cell sorter (FACS) analysis. Ctrl, control 
respectively) (Figure 2a). Long-term survival of cells, which were stimulated for only $4 \mathrm{~h}$, thus simulating chemotherapy, were seeded at low density and were left to grow for 7 days was markedly affected (Figure $2 b$ ): pretubulysin and tubulysin inhibit cellular growth at nanomolar concentrations (25/50 nM), whereas taxol (50 nM) only showed slight effects on clonogenic survival. Representative pictures of the well plates are shown on the right side (Figure $2 b$ ). To determine if treated cells are still able to show anchorage-independent growth, a hallmark of invasive transformed cells, cancer cells were again treated for $4 \mathrm{~h}$ or left untreated, but seeded at very low density in soft agar. The formation of colonies was clearly reduced in tubulysin- and pretubulysin-treated cells (Figure 2c). These data are substantiated by the fact that pretubulysin induces anoikis, a form of cell death characterized by detachment of cells from extracellular matrix (Figure 2d). Anoikis resistance is characteristic for metastatic tumor cells enabling to survive in the detached state and finally to invade into tissues and organs. As shown in Figure 2d, pretubulysin potently kills MDA-MB-231 breast cancer cells kept in a detached state by seeding them on poly-HEMA (poly(2-hydroxyethyl methacrylate))-coated plates.

Pretubulysin induces apoptosis. To analyze whether the diminished proliferation rate of tubulysin- or pretubulysintreated cancer cells is caused by enhanced cell death rates, apoptosis assays were conducted. Both tubulysin A and pretubulysin induced apoptosis in a dose-dependent manner in T24 and MDA-MB-231 cells after treatment for $48 \mathrm{~h}$ (Figure 3a). Pretreatment of cells with the pancaspase inhibitor QVD for $2 \mathrm{~h}$ resulted in decreased apoptosis and argues for a role of caspases both in tubulysin- and in pretubulysin-induced cell death (Figure $3 \mathrm{~b}$ ). Along this line, treatment with tubulysin $A$ and pretubulysin for $48 \mathrm{~h}$ leads to activation of caspase- 8 and -3 in addition to cleavage of Bid and poly(ADP-ribose)-polymerase 1 (PARP), as determined by western blot experiments (Figure $3 \mathrm{c}$ ). Bax activation,
T24

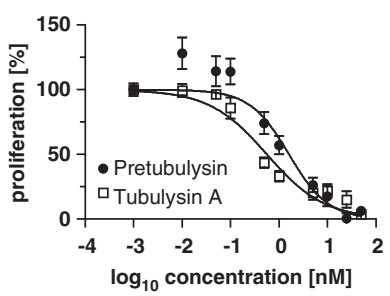

b

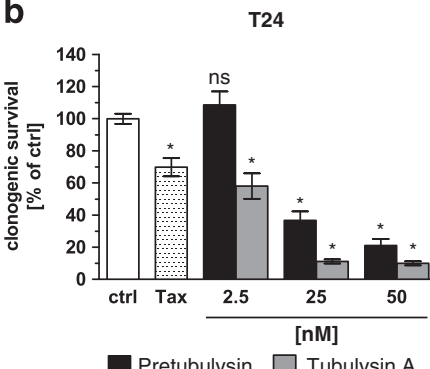

Pretubulysin $\square$ Tubulysin A

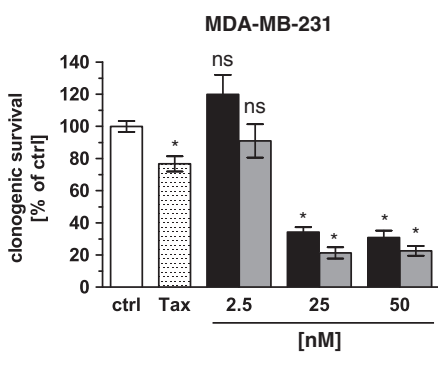

Pretubulysin $\square$ Tubulysin A
MDA-MB-231

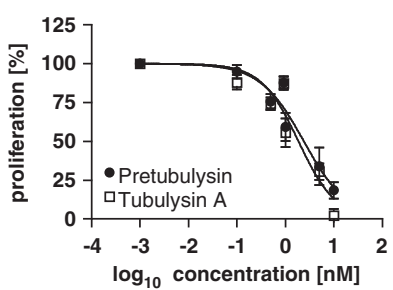

control

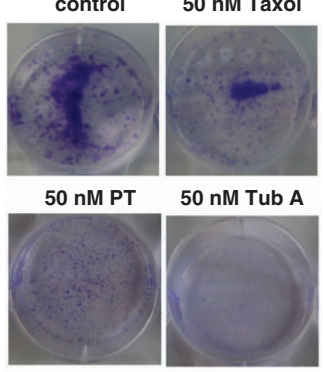

control $\quad 50 \mathrm{nM}$ Taxol

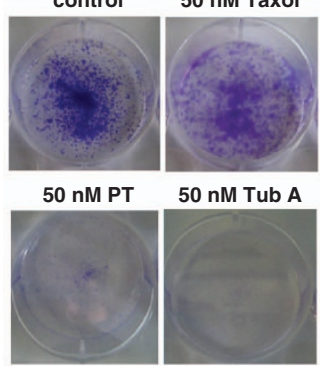

T24
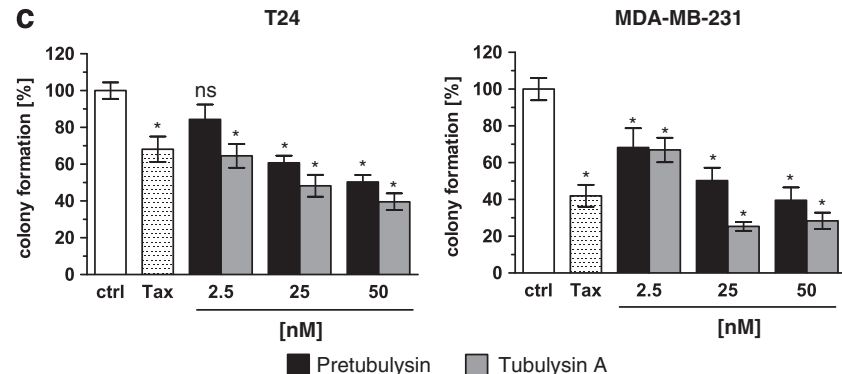

d

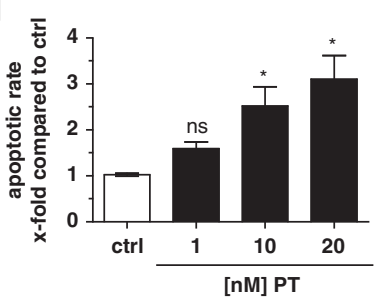

Figure 2 Pretubulysin (PT) and tubulysin A (Tub A) abrogate cell proliferation, long-term survival, anchorage-independent growth and induce anoikis. (a) T24 and MDA-MB-231 cells were treated with PT or Tub A for $72 \mathrm{~h}$. As shown by crystal violet staining, PT inhibits cell proliferation similar to Tub A. (b) PT and Tub A impede clonogenic survival of cancer cells. T24 and MDA-MB-231 cells were treated with PT/Tub A for $4 \mathrm{~h}$, detached, freshly seeded at very low density (5000 cells/6-well plate) and grown for 7 days. Taxol (Tax, $50 \mathrm{nM}$ ) was used as positive control. Representative pictures of wells are shown on the right. (c) PT inhibits anchorage-independent growth of T24 and MDA-MB-231 cells. After treatment with PT/Tub A for $4 \mathrm{~h}$, cells were seeded in very low cell number in soft agar, allowed to grow for several days and stained with MTT. Number of colonies was counted. Treatment with taxol was used as positive control. (d) Increasing concentrations of PT dose-dependently induce anoikis in MDA-MB-231 breast cancer cells after incubation for $48 \mathrm{~h}$. Bars represent the mean \pm S.E.M. of three independent experiments performed in triplicate, ${ }^{\star} P<0.05$ (one-way analysis of variance (ANOVA), Dunnett's test). Ctrl, control; NS, nonsignificant 
measured by FACS analysis, and release of cytochrome $c$ from mitochondria after treatment with the microtubuledisrupting agents led to conclude that the intrinsic apoptotic pathways are involved in cell death induction (Figures 3d and e).

Pretubulysin inhibits tumor growth and metastasis in vivo. The effects of pretubulysin in growth inhibition seen in vitro are underlined and extended by an in vivo chicken chorioallantoic membrane (CAM) model, showing that $10 \mathrm{nM}$ pretubulysin significantly inhibited tumor growth of T24 cells after implantation onto the chorioallantoic membrane of chicken embryos (Figure 4a). Representative images of hematoxylin/eosin-stained sections of the CAM illustrate the growth inhibitory effect of pretubulysin (Figure 4b). Moreover, an in vivo xenograft mouse model was performed, in which a

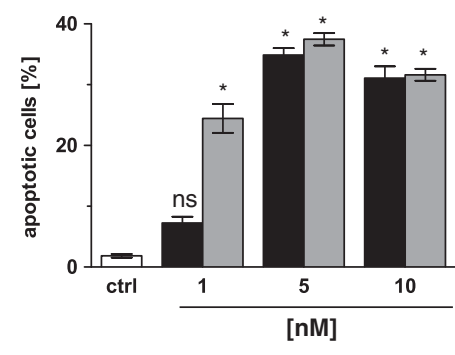

Pretubulysin
MDA-MB-231

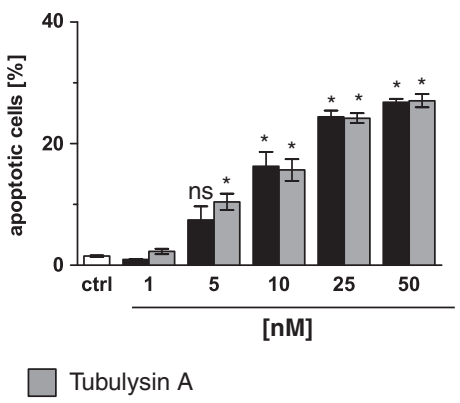

MDA-MB-231

b

T24
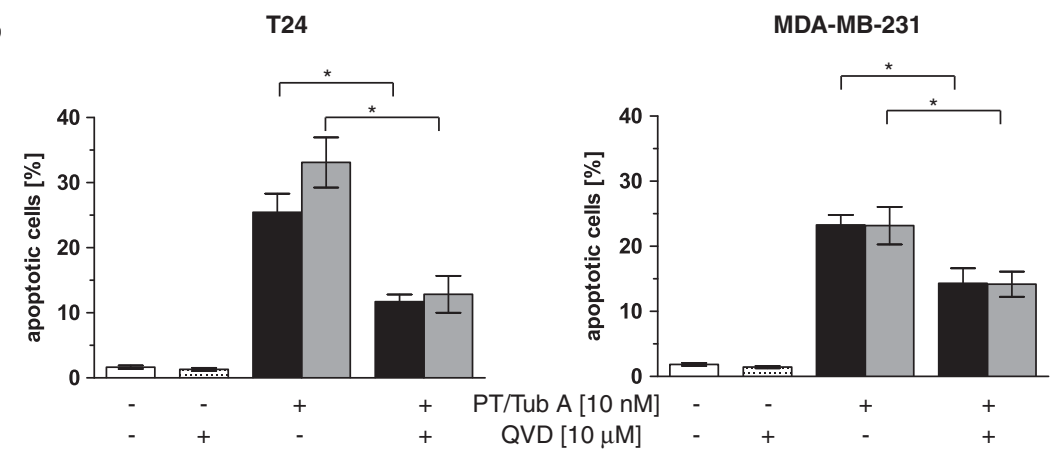

Pretubulysin

Tubulysin A
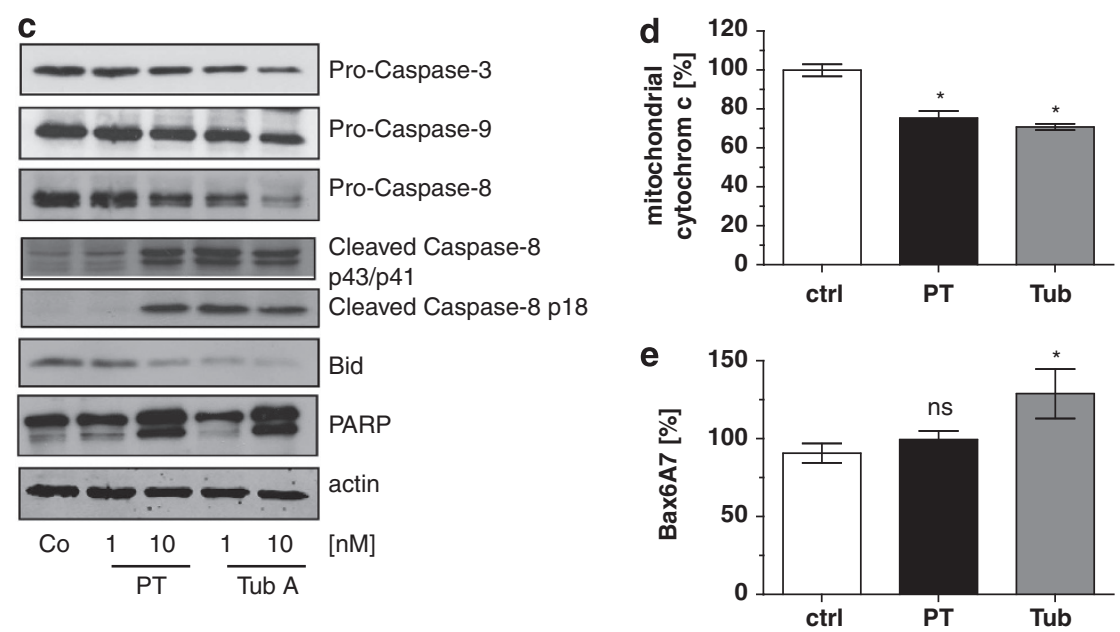

Figure 3 Pretubulysin (PT) and tubulysin A (Tub A) activate the intrinsic apoptosis pathway. (a) Treatment of T24 and MDA-MB-231 cells with PT and Tub A for 48h revealed a significant induction of apoptosis. (b) Pretreatment of T24 and MDA-MB-231 for $2 \mathrm{~h}$ with the pancaspase inhibitor QVD-OPh followed by treatment with PT/Tub A for $48 \mathrm{~h}$ significantly blocked apoptosis induced by PT/Tub A. (c) Western blot analysis showed diminished expression of Bid, procaspase- 9 and -3 and enhanced cleavage of caspase-8 and PARP after treatment with PT and Tub A for $48 \mathrm{~h}$. (d) As monitored by flow cytometry, the amount of mitochondrial cytochrome $c$ is significantly lower in PT/Tub A-treated T24 cells compared with controls. (e) T24 cells were treated for $48 \mathrm{~h}$ with PT/tubulysin, harvested and stained with a specific antibody for the active form of Bax (Bax6A7) and analyzed via flow cytometry. Bars represent the mean \pm S.E.M. of three independent experiments performed in triplicates, ${ }^{*} P<0.05$ (one-way analysis of variance (ANOVA), Dunnett's test). Ctrl, control 
MDA-MB-231 breast cancer cells were subcutaneously inoculated in severe-combined immunodeficiency (SCID) mice. When palpable tumors have been developed, mice were treated with $0.1 \mathrm{mg} / \mathrm{kg}$ pretubulysin every second day for 4 weeks. As shown in Figures $4 c$ and $d$, tumor volume and tumor weight is significantly reduced in pretubulysintreated mice compared with vehicle control-treated mice. In addition, immunohistological stainings showed significantly lower amounts of Ki-67-positive proliferating cells (Figure 4e) and upregulation of apoptosis in pretubulysin-treated mice compared with control mice, as shown by a TdT-mediated dUTP-biotin nick-end labeling (TUNEL) assay (Figure 4f).

Pretubulysin not only inhibits growth of breast tumor cells but also breast cancer cell dissemination into lungs as observed by use of the 4T1-Luc metastatic mouse model. ${ }^{22}$ Intravenously injected 4T1 cells expressing a luciferase reporter allow monitoring the localization of cells by life imaging. In animals pretreated with pretubulysin, 4T1-Luc cells were clearly reduced in their metastatic potential and showed less metastatic lesions in the lungs compared with untreated animals (Figures $4 \mathrm{~g}$ and $\mathrm{h}$ ). Even the lung weight was also significantly affected by pretubulysin treatment (Figure 4i).

Pretubulysin enhances the proapoptotic activity of TRAIL. Both tubulysin and pretubulysin significantly decrease protein expression of the antiapoptotic proteins $\mathrm{Mcl}-1$ and $\mathrm{Bcl}-\mathrm{x}_{\mathrm{L}}$ after $48 \mathrm{~h}$ shown by western blot analysis (Figure 5a). Recent findings indicate that resistance toward TRAIL-induced apoptosis is mainly due to an enhanced expression of the Bcl-2 family member $\mathrm{Mcl}-1$ in different kinds of cancer $^{21}$ and lead to the hypothesis that a combination of tubulysin/pretubulysin with TRAIL might have synergistic effects on induction of cell death. Indeed, when T24 and MDA-MB-231 cells were treated with $1 \mathrm{nM}$ tubulysin A or pretubulysin in combination with $25 \mu \mathrm{M}$ TRAIL, synergistic apoptosis induction was seen as compared with single treatments (Figure $5 b$ ) and was even more obvious in cells treated with $10 \mathrm{nM}$ pretubulysin (Figure $5 \mathrm{c}$ ). Combination of tubulysin A or pretubulysin with TRAIL also displays markedly augmented effects on the activity of pivotal cell death proteins such as caspase-8, Bid or cleaved PARP as shown in Figure $5 \mathrm{~d}$. The chemosensitizing effect of pretubulysin could also be shown in a three-dimensional tumor cell model using T24 cancer cells grown as spheroids, as small concentrations of pretubulysin together with TRAIL strongly enhance the cell death rate compared with TRAIL or pretubulysin treatment alone (Figure 5e).

Pretubulysin regulates the proteasomal degradation of Mcl-1 via Fbw7. After having shown that pretubulysin severely counteracts the insensitivity of the cancer cells to TRAIL-induced apoptosis most likely via inhibition of Mcl-1 expression, we further aimed to unravel the molecular mechanism leading to diminished expression of $\mathrm{Mcl}-1$ protein levels. Neither in the breast nor in the bladder carcinoma cells mRNA expression of $\mathrm{Mcl}-1$ is significantly modified after treatment with pretubulysin for $24 \mathrm{~h}$ (Figure 6a). However, combined treatment with the panprotease inhibitor MG-132 rescues the pretubulysin-mediated downregulation of $\mathrm{Mcl}-1$
(Figure 6b), indicating a role of the ubiquitin-proteasome pathway in this matter. Previous studies identified the SCFE3 ubiquitin ligase as an important regulator of $\mathrm{Mcl}-1$ protein levels. The F-box protein Fbw7 is the substrate recognition component of this complex that targets proteins such as $\mathrm{Mcl}-1$ for ubiquitination and destruction. ${ }^{23,24}$ In fact, we were able to demonstrate that inhibition of Fbw7 protein expression by specific siRNAs resulted in enhanced protein levels of $\mathrm{Mcl}-1$ in MDA-MB-231 breast cancer cells (Figure 6c). As it is known that Fbw7-mediated degradation of Mcl-1 depends on its phosphorylation, ${ }^{25}$ we analyzed the expression of $\mathrm{p}-\mathrm{Mcl}-1$ in pretubulysin-treated cells and observed an enhanced phosphorylation status of Mcl-1 after $24 \mathrm{~h}$ (Figure 6d). Searching for the upstream signaling pathway responsible for the effect of pretubulysin on Mcl-1 phosphorylation and degradation revealed a dose-dependent activation of p38 and JNK mitogen-activated protein kinase cascades in MDA-MB-231 cells incubated with pretubulysin (Figure 6d). Interestingly, treatment with the p38 inhibitor SB203580 for $24 \mathrm{~h}$ had no effect on the expression level of $\mathrm{Mcl}-1$, whereas specific inhibition of the JNK pathway using SP600125 led to an upregulation of $\mathrm{Mcl}-1$ expression. In addition, while combined treatment of the cells with the p38 inhibitor and pretubulysin did not influence the expression of $\mathrm{Mcl}-1$, incubation of the cells with pretubulysin in combination with the JNK inhibitor counteracts pretubulysin-mediated degradation of $\mathrm{Mcl}-1$, as demonstrated by western blot experiments (Figure 6e). Finally, apoptosis assays demonstrate that pretubulysin is not able to induce apoptosis in our cancer cell lines when the JNK pathway is blocked by a specific inhibitor, whereas the p38 signaling seems not to be involved in pretubulysin-mediated cytotoxicity (Figure 6f). These results strongly imply that the JNK pathway has an important role in the signaling cascade resulting in destruction of $\mathrm{Mcl}-1$.

\section{Discussion}

The tubulin network is a very appealing and important therapeutic target in treatment of various cancer types including numerous hematological malignancies and solid tumors. The development of microtubule-stabilizing agents, which comprise taxanes and epothilone, and microtubuledestabilizing drugs, such as the plant alkaloids vinblastine and vincristine, gave new hope in the struggle against cancer when administrated either alone or in combination regimens with chemotherapeutic agents and are now well accepted in the clinic. ${ }^{26}$ These microtubule-targeting agents are derived from natural sources and display complex structural features challenging their chemical synthesis and thus their supply. ${ }^{26}$ Other major drawbacks of these potent antimitotic cancer drugs are the occurrence of resistance mechanisms ${ }^{27}$ and the high risk of neurotoxicity in patients treated with antitubulin agents. ${ }^{28}$ Accordingly, new compounds targeting the microtubule network that overcome these problems are urgently needed.

The group of Sasse and co-workers ${ }^{29}$ isolated in 2000 a new class of complex peptides from myxobacteria named tubulysins. Tubulysins bind at the vinca domain of $\beta$-tubulin, thus disrupting microtubule assembly. ${ }^{30}$ Unfortunately, despite their very promising anticancer impact - tubulysins 
show a 20 - to 100 -fold enhanced growth inhibition potential compared with taxol, epothiolone and vincristine, exhibit antiangiogenic activities and retain cytotoxic effects even in multidrug-resistant cells lines - clinical development of these natural products is hampered because of their very poor supply and their challenging synthesis pathway. ${ }^{29,31}$ a

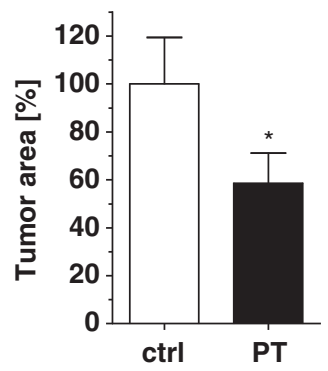

C

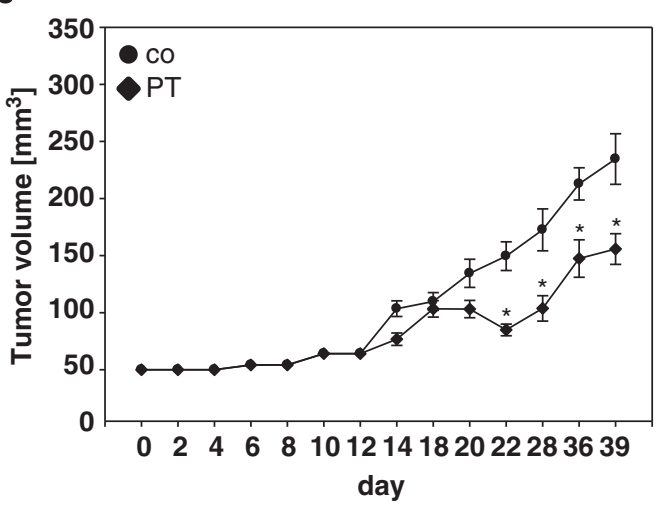

e

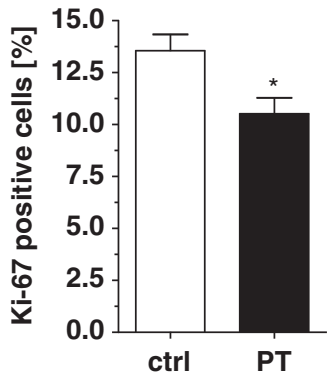

Ki-67

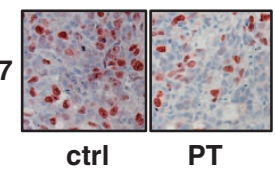

g

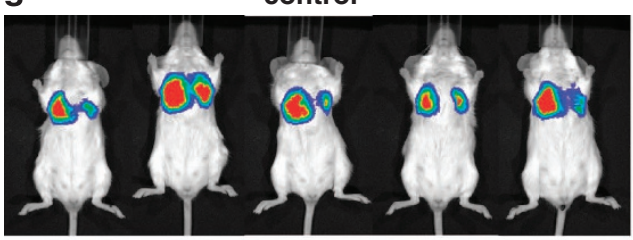

Pretubulysin $[0.1 \mathrm{mg} / \mathrm{kg}]$

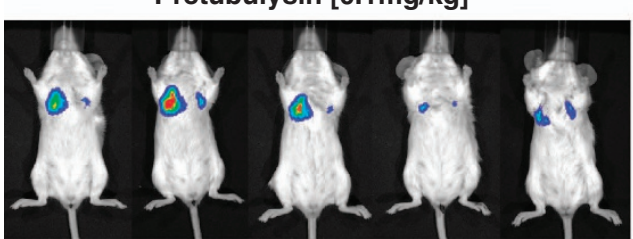

b

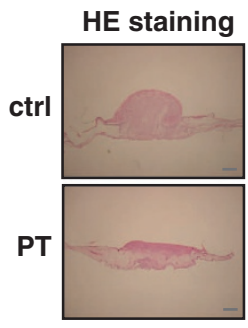

d
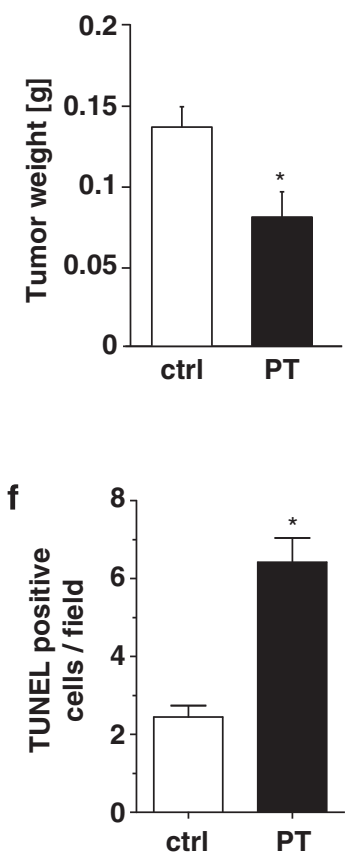

TUNEL

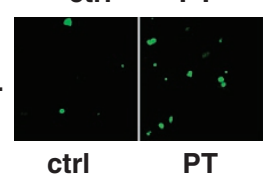

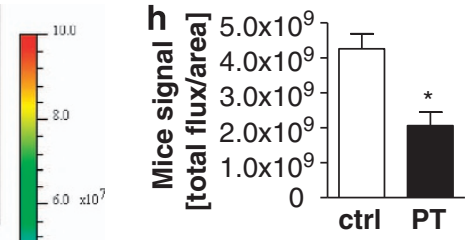

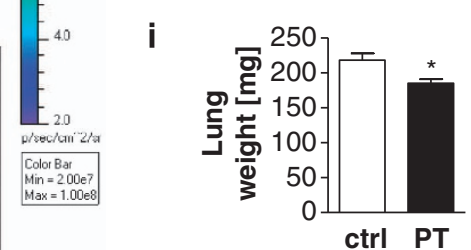


This study sets the stage for pretubulysin, a stable precursor of the tubulysins that is less reactive than its parental substances, and most importantly, chemically accessible, thus circumventing the downsides of supply typical for microtubule-targeting agents. ${ }^{17}$ Extending the initial study of Herrmann et al. ${ }^{18}$ showing that pretubulysin inhibits tubulin assembly in vitro, induces apoptosis and abrogates migration of cancer cells, the here presented in vitro and in vivo assays clearly demonstrate that pretubulysin impedes tumor growth and metastasis equally potent to the already established but chemically not available antimitotic compound tubulysin.

Pretubulysin affects multiple processes implicated in cancer growth and metastasis, namely proliferation, longterm survival, anchorage-independent growth, anoikis and apoptosis induction by activation of caspases, Bid and Bax, cytochrome $c$ release and degradation of the antiapoptotic protein Mcl-1. Next to its anticancer effect in single-agent treatment, pretubulysin strongly sensitizes to TRAIL-induced apoptosis, and thus broadens its therapeutic potential as a lead substance for novel antimitotic drugs in treatment of metastatic cancer.

As TRAIL induces apoptosis selectively in transformed cells without influencing healthy cells, it is an attractive drug in treatment of cancer, and clinical trials of TRAIL and agonistic TRAIL receptor antibodies are currently under way. ${ }^{32}$ Nevertheless, it was shown that several tumors are resistant to TRAIL. One major player in TRAIL resistance is $\mathrm{Mcl}-1^{21}$ steering our working hypothesis that pretubulysin has chemosensitizing effects to TRAIL-induced apoptosis, which in fact has been proven in two- and three-dimensional cell culture assays. Our data are supported by the study of Sanchez-Perez et al. ${ }^{33}$ demonstrating that by inducing mitotic arrest and degradation of antiapoptotic proteins, the microtubule-interfering agents nocodazole and taxol sensitize to TRAIL-mediated cytotoxicity.

Although numerous studies already confirmed that cancer cells undergo apoptosis in response to microtubule-targeting agents, the molecular signaling cascades underlying the induction of cell death are not yet examined in detail. ${ }^{28}$ We used pretubulysin as a chemical tool to unravel the mechanism of microtubule-destabilizing agents leading to cytotoxicity. The work of Wertz et al. ${ }^{34}$ gave interesting hints on how antimitotic agents are able to induce cell death. The authors showed that the expression of the prosurvival protein $\mathrm{Mcl}-1$ is diminished in cells arrested in mitosis. Stress-activated kinases and phosphatases coordinate the inactivation of
$\mathrm{Mcl}-1$ by the ubiquitin ligase complex $\mathrm{SCF}^{\mathrm{Fbw} 7}$, which finally results in its proteasomal degradation. ${ }^{34}$ These findings were confirmed and extended by our own work. Treatment of breast and bladder carcinoma cells with pretubulysin or tubulysin abrogates the protein expression of $\mathrm{Mcl}-1$, whereas mRNA levels are not affected. Consistent with the study of Inuzuka et al., ${ }^{25}$ inhibition of Fbw7 expression via siRNAs stabilizes the protein levels of $\mathrm{Mcl}-1$. Fbw7 is the substrate recognition part of the SCF ubiquitin ligase, which is involved in targeting several oncoproteins, including Mcl-1, c-Myc, c-Jun and Notch, for ubiquitylation and degradation via recognition of a consensus phosphorylated degron sequence. ${ }^{23}$ Indeed, pretubulysin induces phosphorylation of Mcl-1 most likely by activation of $\mathrm{JNK}$, but not of $\mathrm{p38}$, both kinases were known to interact with $\mathrm{Mcl}-1 .{ }^{34}$ Previous studies demonstrated that microtubule-interacting agents activate JNK signaling pathways. Intracellular stress caused by microtubule-targeting substances results in activation of Ras, ASK1 (apoptosis signal-regulating kinase 1) and SEK1 (stress-activated protein kinase 1) signaling, which leads to an activation of JNK. ${ }^{35,36}$ As inhibition of JNK but not of p38 signaling (by use of specific inhibitors) neutralizes the cytotoxic effect of pretubulysin, we gained a pretty clear picture how microtubule-destabilizing agents such as pretubulysin evoke apoptosis: JNK-dependent phosphorylation of $\mathrm{Mcl}-1$ directs this antiapoptotic protein toward proteasomal degradation via $\mathrm{SCF}^{\mathrm{Fbw}}$. The knowledge on the molecular signaling of pretubulysin provides a rational basis for combination therapies in metastatic cancer.

In summary, we characterized the microtubule-destabilizing agent pretubulysin as a simplified chemical accessible tubulysin derivative with anticancer effects both in single-agent treatment and in combination with TRAIL in an equal potency as the parent substance tubulysin. Pretubulysin has proven to be both a valuable chemical tool to investigate the mode of action of microtubule-destabilizing agents and a promising lead for the development of antimetastatic drugs.

\section{Materials and Methods}

Compounds and antibodies. Pretubulysin (chemical structure is depicted in Supplementary Figure S1) was synthesized as described previously. ${ }^{17}$ Tubulysin A was from Merck Calbiochem (Darmstadt, Germany). Both compounds were dissolved in dimethyl sulfoxide (DMSO) in high concentrated stocks. Further dilutions were carried out in culture media or PBS. SuperKillerTRAIL was purchased from Enzo Life Sciences (Farmingdale, NY, USA). Apo2L/TRAIL was kindly provided by Genentech (South San Francisco, CA, USA). The caspase inhibitor QVD-OPh was purchased from R\&D Systems (Wiesbaden, Germany). Propidium iodide and Hoechst 33258 were obtained from Sigma-Aldrich

Figure 4 Pretubulysin (PT) inhibits tumor growth and metastasis in vivo. (a and $\mathbf{b})$ CAM model. T24 cells were seeded on the CAM of chicken embryos and treated with $10 \mathrm{nM} \mathrm{PT}$ or DMSO for 3 days. Tumor growth was determined using hematoxylin and eosin (HE)-stained paraffin sections of the CAM as described in Materials and Methods. (a) Analysis of tumor area, described as percentage of the untreated control group with mean \pm S.E.M. of three independent experiments $\left({ }^{*} P<0.05\right)$, demonstrates the growth inhibitory impact of PT. (b) Representative pictures of hematoxylin and eosin-stained sections of the CAM are shown; scale bar: $500 \mu \mathrm{m}$. (c-f) In vivo xenograft model. MDA-MB-231 breast cancer cells were injected subcutaneously in SCID mice. When tumors had established, mice were treated with $0.1 \mathrm{mg} / \mathrm{kg}$ PT every second day. (c) Tumor volume as well as (d) tumor weight was significantly reduced in PT-treated mice compared with control animals. (e) Staining of tumor tissues of PT-treated mice revealed significantly diminished Ki-67-positive cells in comparison with control mice; ${ }^{*} P \leq 0.05$ (one-way analysis of variance (ANOVA) Holm-Sidak). (f) TUNEL staining demonstrated an increased number of apoptotic cells per microscopic field in PT-treated mice in comparison with controls. ( $\mathbf{g}-\mathbf{i})$ In vivo cancer metastasis model. BALB/cByJRi mice were pretreated with $0.1 \mathrm{mg} / \mathrm{kg}$ PT or solvent control. (g) Bioluminescence signals were recorded by imaging dorsoventral and ventrodorsal sides of the mice on day 8 after intravenous injection of 4T1-Luc cells intravenously. Five representative mice out of 8 or 10 treated animals, respectively, are shown. (h) Luciferase signals were calculated as photons $/ \mathrm{s} / \mathrm{cm}^{2}$ (total flux/area). (i) The weight of the lungs isolated from PT-treated BALB/cByJRj mice on day 8 is significantly reduced compared with lung weights of control mice; ${ }^{*} P \leq 0.05 t$-test. Ctrl, control 
a

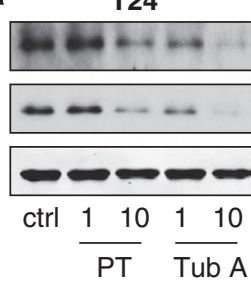

MDA-MB-231

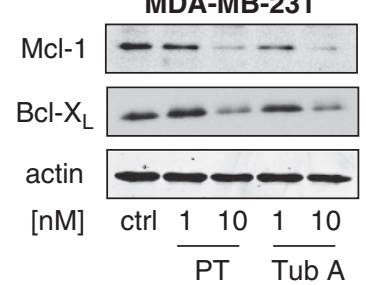

b

T24

Pretubulysin

$\square$ Tubulysin A

MDA-MB-231

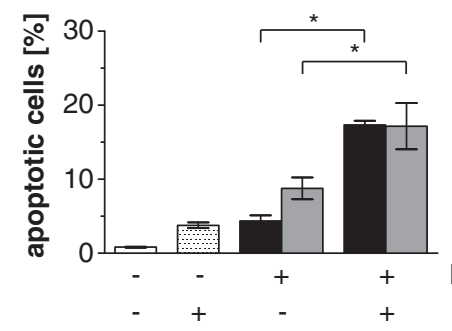

PT/Tub A [10 nM]

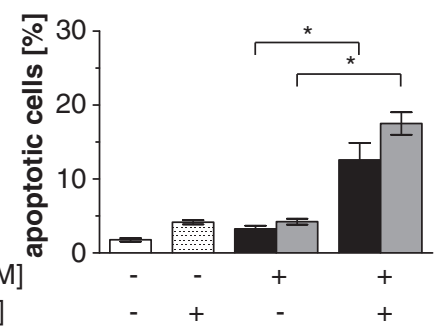

c

T24

Pretubulysin $\square$ Tubulysin A

MDA-MB-231

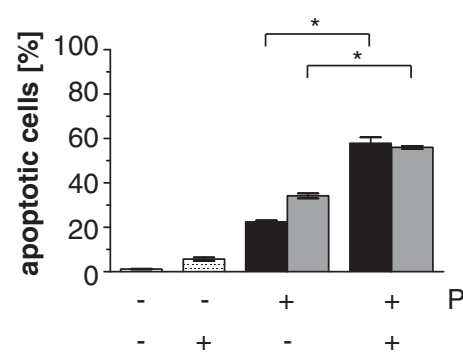

PT/Tub A [10 nM] TRAIL [25 $\mu \mathrm{M}]$

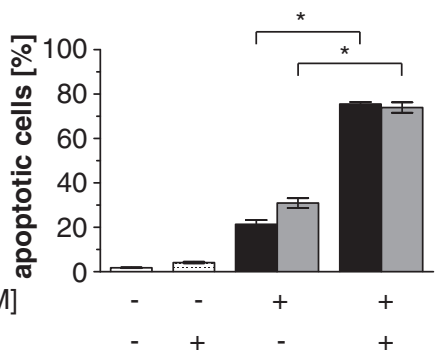

d

T24

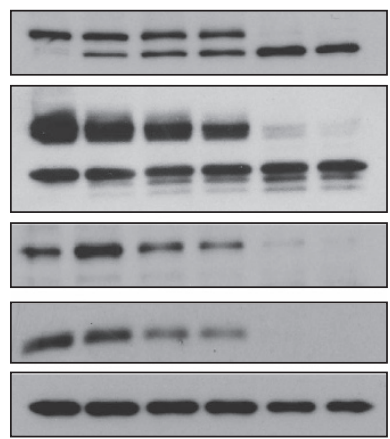

PARP

Pro-Caspase-8

$\leftarrow \mathrm{p} 43 / \mathrm{p} 41 \rightarrow$

$\mathrm{Mcl}-1$

Bid

$\beta$-actin

- $\quad$ PT Tub PT Tub PT/Tub A[10nM]

$-\quad+\quad-\quad+\quad+\quad$ TRAIL $[25 \mu \mathrm{M}]$

MDA-MB-231
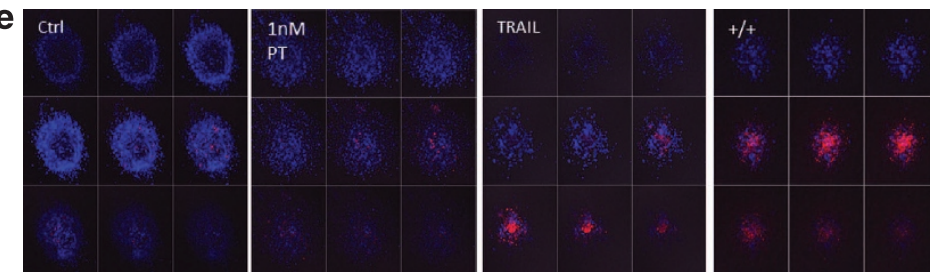

Hoechst

propidium

iodide

Figure 5 Pretubulysin (PT) sensitizes to TRAlL-induced cytotoxicity. (a) Treatment of T24 and MDA-MB-231 cells with PT and tubulysin dose-dependently abrogates the protein expression of Mcl-1 and Bcl- $\mathrm{x}_{\mathrm{L}}$ after $48 \mathrm{~h}$, as shown by western blot. (b) PT/tubulysin A (Tub A) enhances the proapoptotic activity of TRAIL synergistically. T24/MDAMB-231 cells were treated for $48 \mathrm{~h}$ with TRAIL plus $1 \mathrm{nM}$ PT/Tub A or (c) $10 \mathrm{nM} \mathrm{PT/Tub} \mathrm{A,} \mathrm{respectively.} \mathrm{(d)} \mathrm{TRAIL} \mathrm{increases} \mathrm{the} \mathrm{effect} \mathrm{of} \mathrm{PT} \mathrm{on} \mathrm{protein} \mathrm{expression} \mathrm{of} \mathrm{PARP,}$ caspase-8, Mcl-1 and Bid, as shown by western blot. (e) Intraspheroidal cell death is strongly augmented in spheroids treated for 4 days with TRAIL in combination with $1 \mathrm{nM}$ PT compared with each drug alone. Representative images of $z$-stacks, each with a distance of $20 \mu \mathrm{m}$, are shown. Bars represent the mean \pm S.E.M. of three independent experiments performed in triplicate; ${ }^{*} P<0.05$ (one-way analysis of variance (ANOVA), Dunnett's test). Ctrl, control 
a

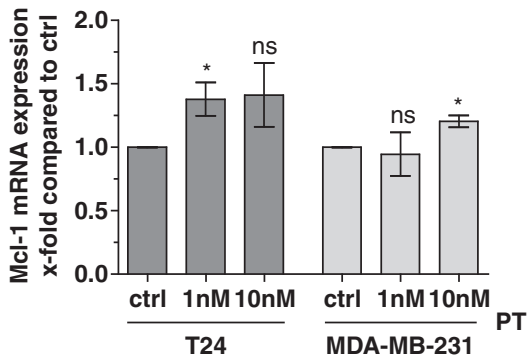

C

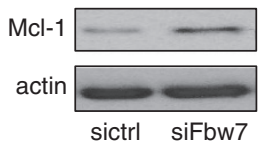

e

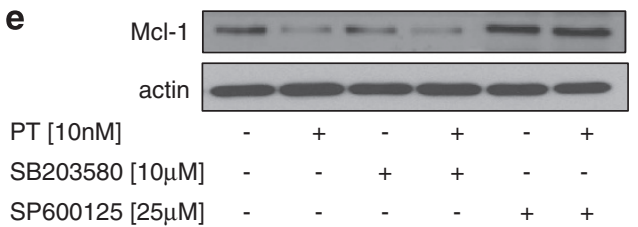

b

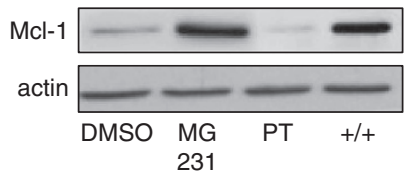

d

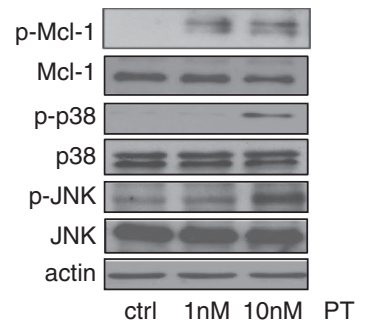

f

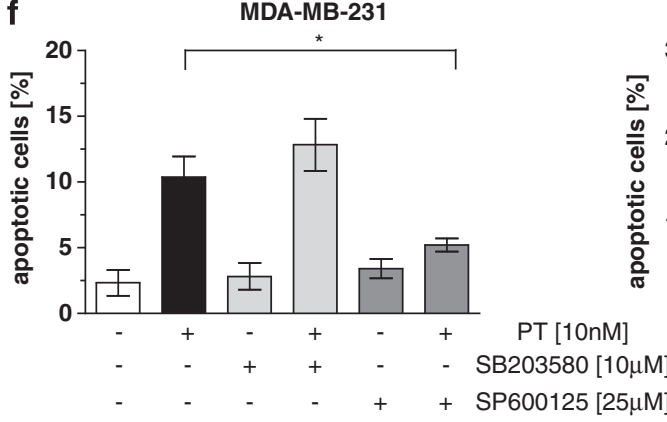

T24

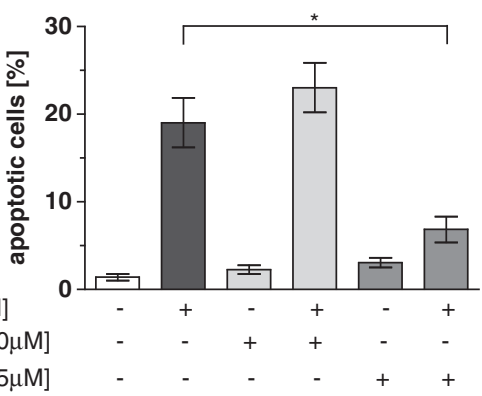

Figure 6 Mechanism of pretubulysin (PT)-mediated downregulation of Mcl-1. (a) Real-time PCR analysis revealed that treatment of T24 and MDA-MB-231 cells with PT has no significant impact on mRNA expression of Mcl-1. (b) Inhibition of the proteasome by MG-132 counteracts the PT -mediated decrease of Mcl-1 protein levels. (c) Transfection of MDA-MB-231 cells with specific small interfering RNAs (siRNAs) against Fbw7 results in enhanced protein expression of Mcl-1. (d) Phosphorylation of Mcl-1, p38 and JNK is increased in PT-treated MDA-MB-231 cells after $24 \mathrm{~h}$ compared with DMSO-treated cells. Total Mcl-1, p38, JNK and actin levels served as control. (e) Inhibition of p38 pathways by $10 \mu \mathrm{M}$ of the specific p38 inhibitor SB203580 had no influence on Mcl-1 expression, whereas treatment with $25 \mu \mathrm{M}$ of the JNK inhibitor SP600125 results in clear upregulation of Mcl-1 expression in MDA-MB-231 cells. Combined treatment of the cells with the JNK inhibitor and PT stabilizes the protein level of Mcl-1. (f) MDA-MB-231 and T24 cells were pretreated for $18 \mathrm{~h}$ with $10 \mu \mathrm{M}$ SB203580 or $25 \mu \mathrm{M}$ SP600125 following incubation with $10 \mathrm{nM}$ PT for $48 \mathrm{~h}$. Fluorescence-activated cell sorter (FACS) analysis demonstrated that PT-induced apoptosis is not affected by inhibition of p38, whereas blocking JNK pathways is sufficient to abrogate apoptosis in PT-treated cells. ${ }^{\star} P<0.05$ (one-way analysis of variance (ANOVA), Dunnett's test). Ctrl, control

(Taufkirchen, Germany). The primary antibodies PARP, $\beta$-tubulin, caspase-9, caspase-8, Mcl-1, Bcl- $\mathrm{x}_{\mathrm{L}}$, cytochrome $c, \mathrm{p}-\mathrm{p} 38, \mathrm{p} 38$, JNK and $\mathrm{p}-\mathrm{JNK}$ were from Cell Signaling Technology (Danvers, MA, USA). The $\alpha$-tubulin antibody was obtained from Abcam (Cambridge, UK), the actin antibody was from Merck Millipore (Billerica, MA, USA), p-Mcl-1 antibody was purchased from Bioss Inc. (Woburn, MA, USA) and the caspase- 3 antibody and the antibody for the active conformation of Bax/Bax6A7 were purchased from BD Biosciences (San Jose, CA, USA). The following secondary antibodies were used: goat-anti-rabbit-488, goat-anti mouse-488 (AlexaFluor; Life Technologies, Darmstadt, Germany), goat-anti-rabbit (Dianova, Hamburg, Germany), goat-anti-mouse IgG2a (SouthernBiotech, Birmingham, AL, USA), goat-anti-mouse IgG2b (SouthernBiotech), goat-anti-mouse IgG1 (Biozol, Eching, Germany). Phalloidin-rhodamine was purchased from Sigma-Aldrich.

Cell culture. The human urinary bladder carcinoma cell line T24 was kindly provided by Barbara Mayer (Department of Surgery, University of Munich, Munich, Germany) and recently authentized by the DSMZ (Braunschweig, Germany). T24 were maintained in McCoy's medium (PAA, Coelbe, Germany) supplemented with $10 \%$ heat-inactivated fetal calf serum (FCS; Biochrom AG, Berlin, Germany), $1 \%$ glutamine and $0.1 \%$ penicillin/streptomycin (PAA). MDA-MB-231 cells were obtained from Cell Line Services (CLS, Eppelheim, Germany) and were grown in DMEM medium (PAA) supplemented with 10\% FCS and $0.1 \%$ penicillin/streptomycin. 4T1-Luc cells were purchased from Caliper Life Sciences (Hopkinton, MA, USA) and cultivated in RPMI (PAA) with 10\% FCS (Biochrom AG).

Microtubule staining. T24 and MDA-MB-231 cells were treated with pretubulysin or tubulysin A for 24 or $16 \mathrm{~h}$, respectively. Cells were washed two times and the extraction buffer was added $(5 \times$ concentrated: $400 \mathrm{mM}$ PIPES, $5 \mathrm{mM} \mathrm{MgCl}_{2}, 5 \mathrm{mM}$ EGTA, 0.5\% Triton). After 2-min incubation, $5 \%$ glutaraldehyde was added for another $10 \mathrm{~min}$. Suspension was discarded and a $0.1 \% \mathrm{NaBH}_{4} /$ PBS solution was added for 7 min. Cells were washed three times with PBS, blocked for $10 \mathrm{~min}$ with $0.2 \%$ BSA/PBS and incubated for $30 \mathrm{~min}$ with anti $\alpha$-tubulin antibody. After further washing steps, samples were incubated for $30 \mathrm{~min}$ with the secondary antibody and Hoechst 33258. Subsequently, cells were prepared for confocal microscopy. 
Measurement of apoptotic cell death and cell cycle analysis. Quantification of apoptotic cell death was performed as described by Nicoletti et al. ${ }^{37}$ Briefly, cells were permeabilized using a Triton X-100-containing buffer and stained with $50 \mu \mathrm{g} / \mu \mathrm{l}$ propidium iodide. Cells were analyzed by flow cytometry (FACSCalibur; Becton Dickinson, Heidelberg, Germany). Cell cycle was analyzed by measuring in the linear mode; cell death rates were determined by analyzing the fluorescence in logarithmic mode.

Proliferation assay. Cells were seeded the day before treatment (1500 cells/ 96-well plate) and incubated for $72 \mathrm{~h}$ with pretubulysin or tubulysin $\mathrm{A}$. The media were discarded, cells washed with PBS, stained and fixed with crystal violet/ $0.5 \%$ methanol for $10 \mathrm{~min}$. Crystal violet was redissolved with sodium citrate solution to measure absorption at $550 \mathrm{~nm}$ at a SpectraFluor Plus (Tecan, Männedorf, Austria). For statistical analysis, untreated cells were set to $100 \%$ viable cells.

Clonogenic survival and anchorage-independent growth. Following treatment with pretubulysin or tubulysin A for $4 \mathrm{~h}$, T24 or MDA-MB-231 cells were trypsinized and 5000 cells were seeded in a 6-well plate. Cells were allowed to grow for 10 days and stained with crystal violet for $10 \mathrm{~min}$. Pictures of the wells were taken and absorption was measured at $550 \mathrm{~nm}$ at a SpectraFluor Plus (Tecan). For statistical analysis, untreated cells were set to $100 \%$ viable cells. For colony formation assay, treated cells were seeded in a matrix made out of two layers of soft agarose (bottom layer: $1 \%$ agarose; top layer: $0.4 \%$ agarose). A total of $2.5 \times 10^{5}$ cells were resuspended in $0.5 \%$ agarose and seeded on top of the agarose matrix. Cells were grown for 7-10 days, followed by staining with MTT (3-[4,5-dimethylthiazol-2-yl]-2,5 diphenyl tetrazolium bromide). The number of colonies was counted.

Detachment-induced anoikis assay. MDA-MB-231 cells $\left(7 \times 10^{4}\right)$ were kept in suspension by maintaining them on poly-HEMA (Sigma-Aldrich)-coated culture dishes. To prevent cellular clumping, medium was supplemented with $1 \%$ methylcellulose (Sigma-Aldrich) to increase viscosity. At $24 \mathrm{~h}$ after seeding, cells were stimulated with indicated concentrations of pretubulysin for $48 \mathrm{~h}$. Cell death was analyzed as described above.

Western blot. Proteins were separated by SDS-PAGE and transferred to nitrocellulose membranes using tank blotting. For the detection of protein levels, the ECL Prime Western Blotting Detection System (GE Healthcare, Buckinghamshire, UK) or the Odyssey Infrared Imaging system version 2.1 (LI-COR Biosciences, Lincoln, NE, USA) was used.

Analysis of Bax activation. Cells were harvested and fixed with 0.5\% PFA for $30 \mathrm{~min}$ on ice. Cells were washed with PBS/1\% FCS before staining with $0.5 \mu \mathrm{g}$ anti-Bax6A7 antibody (BD Bioscience) in a buffer containing $1 \% \mathrm{FCS}$, $50 \mu \mathrm{g} / \mathrm{ml}$ digitonin in PBS for $1 \mathrm{~h}$. After washing, cells were incubated with the secondary antibody diluted in the same buffer for $30 \mathrm{~min}$ in the dark. After another washing step, active Bax was measured by flow cytometry.

Cytochrome $c$ release. Treated cells were harvested and incubated in a digitonin-containing buffer for $30 \mathrm{~min}$ on ice $(0.2 \mathrm{mM} \mathrm{Na}-\mathrm{EGTA}, 100 \mathrm{mM} \mathrm{KCl}$, $50 \mu \mathrm{g} / \mathrm{ml}$ digitonin, PBS). Cells were fixed with $4 \%$ PFA for $20 \mathrm{~min}$. After washing, samples were blocked ( $3 \% \mathrm{BSA}, 0.05 \%$ saponine) for $1 \mathrm{~h}$ at room temperature and incubated with cytochrome $C$ antibody overnight at $4{ }^{\circ} \mathrm{C}$ with light agitation. The next day, samples were washed with PBS and incubated with the secondary antibody for $1 \mathrm{~h}$. After further washing steps, the amount of cytochrome $c$ in the mitochondria was analyzed by flow cytometry.

Quantitative real-time PCR. Total cellular RNA was isolated and transcribed into cDNA using the RNeasy Mini Kit (Qiagen, Hilden, Germany) and the First-Strand cDNA Kit (Bio-Rad, Munich, Germany). Quantitative real-time PCR was performed on an ABI 7300 RealTime PCR system with TaqMan gene expression Assays (Life Technologies). Calculation of relative mRNA levels was carried out as described by Pfaffl. ${ }^{38}$

Intraspheroidal cell death assay. Spheroids were generated according to the hanging drop method. ${ }^{39}$ After harvesting, spheroids were treated with pretubulysin and/or TRAIL and embedded in collagen. Cells were stained with $125 \mathrm{ng} / \mathrm{ml}$ Hoechst 33258 and $50 \mu \mathrm{g} / \mathrm{ml}$ propidium iodide on days 0 and 4 after treatment for 4 or $1 \mathrm{~h}$, respectively, following confocal laser scanning microscopy using a Zeiss LSM 510 Meta microscope (Zeiss, Jena, Germany). A series of images in z-stack was taken.

Ex vivo and in vivo experiments: CAM assay/tumor growth/ tumor metastasis models. CAM assay was performed as described previously. ${ }^{40}$ Briefly, $1 \times 10^{6}$ T24 cells were resuspended in $10 \mu \mathrm{l} \mathrm{NaCl} 0.9 \%$ (B Braun, Melsungen AG, Germany) and $10 \mu$ l Matrigel matrix (BD Biosciences), implanted on the CAM of fertilized chicken eggs on day 8 of incubation and allowed to form tumors. The next day, tumors were treated with $10 \mathrm{nM}$ pretubulysin or DMSO daily for 3 days. At 4 days after inoculation, tumors were excised with the surrounding CAM, fixed in $4 \%$ paraformaldehyde, embedded in paraffin, cut in $5 \mu \mathrm{m}$ sections and stained with $1: 1$ hematoxylin and $0.5 \%$ eosin solution for histological analysis. Images were digitally recorded at a magnification of 3 with an SZ61 microscope (Olympus, Center Valley, PA, USA) and the tumor area were analyzed using ImageJ digital imaging software $(\mathrm{NIH}$, Bethesda, $M D, U S A)$.

For the subcutaneous xenograft model, $3 \times 10^{6}$ MDA-MB-231 cells in Matrigel/ PBS were injected subcutaneously in the flank of female SCID (C.B-17/lcrHanHsdPrkdc scid) mice, obtained from Janvier (Le Genest-St-Isle, France). According to the previous study of Rath et al., ${ }^{20} 6$ days after tumor cell injection, $0.1 \mathrm{mg} / \mathrm{kg}$ pretubulysin in PBS was injected intravenously every second day. After 39 days, mice were killed and tumor growth and weight of 10 control- and 7 pretubulysintreated mice was determined. Tumor volume was calculated every second day according to the formula: $V=\pi / 6 \times$ length $\times$ width $\times$ height.

For the metastasis model, 20 female BALB/cByJRj mice were purchased from Janvier. Mice were inoculated with $1 \times 10^{5} 4 \mathrm{~T} 1$-Luc cells via the tail vein. At 24 and $4 \mathrm{~h}$ before tumor cell injection, the treatment group $(n=10)$ was medicated with $0.1 \mathrm{mg} / \mathrm{kg}$ pretubulysin (5\% DMSO/PBS) intravenously. On day 8 after tumor cell inoculation, bioluminescence imaging of the mice was performed under anesthesia (2\% isoflurane in oxygen) using the IVIS Lumina system with Living Image software 3.2 (Caliper Life Sciences, Hopkinton, MA, USA) $15 \mathrm{~min}$ after intraperitoneal injection of $6 \mathrm{mg} \mathrm{Na-luciferin} \mathrm{(Promega,} \mathrm{Hilden,} \mathrm{Germany).} \mathrm{Thereafter,} \mathrm{mice} \mathrm{were}$ killed through cervical dislocation and lungs were harvested and weighed. For image analysis, regions of interests were defined and the total signal per ROI was calculated as photons $/ \mathrm{s} / \mathrm{cm}^{2}$ (total flux/area). All pictures were taken with an exposure time of $5 \mathrm{~s}$ and medium binning.

All animal experiments were performed according to the guidelines of the German law for protection of animal life and approved by the local ethics committee.

Immunhistological staining of tumor tissues. Tumor sections were fixed, embedded in paraffin and cut into $5 \mu \mathrm{m}$ slices. Apoptotic cells were determined by staining with ApopTag Fluorescein In Situ Apoptosis Detection Kit (Merck Millipore) according to the manufacturer's instructions. TUNEL-positive cells were visualized and counted using an LSM 510 Meta microscope (Zeiss).

For staining with Ki-67, tumor sections were pretreated with sodium citrate buffer (pH 6.0) for $20 \mathrm{~min}$ at $95^{\circ} \mathrm{C}$ and incubated with Ki-67 antibody (1:100; Abcam; no. 15580) for $1 \mathrm{~h}$ at room temperature. The biotin/avidin interaction system of the VECTASTAIN ABC Kit (Vector Laboratories, Burlingame, CA, USA) was used for detection. Sections were counterstained with hematoxylin and mounted with FluorSave (Merck Millipore). Ki-67-positive cells were counted using an inverted microscope.

Statistical analysis. All experiments were performed at least three times in triplicate. Results are expressed as mean value \pm S.E.M. One-way ANOVA/ Dunnett's test, one-way ANOVA/Holm-Sidak or individual Student's $t$-tests were performed using GraphPad Prism software (GraphPad Software Inc., San Diego, CA, USA). $P$-values $<0.05$ were considered as significant.

\section{Conflict of Interest}

The authors declare no conflict of interest.

Acknowledgements. This work was supported by the Deutsche Forschungsgemeinschaft (DFG), FOR 1406, to AMV, UZ and EW, as well as Cluster of Excellence Nanosystems Initiative Munich (Bioimaging to EW). We thank Florian Förster, Christina Schempp, Rita Socher, Kerstin Loske and Sylvia Schnegg for excellent technical assistance. 
1. Nguyen DX, Bos PD, Massague J. Metastasis: from dissemination to organ-specific colonization. Nat Rev Cancer 2009; 9: 274-284.

2. Mehlen P, Puisieux A. Metastasis: a question of life or death. Nat Rev Cancer 2006; 6 : 449-458.

3. Wyckoff JB, Jones JG, Condeelis JS, Segall JE. A critical step in metastasis: in vivo analysis of intravasation at the primary tumor. Cancer Res 2000; 60: 2504-2511.

4. Wong CW, Lee A, Shientag L, Yu J, Dong Y, Kao G et al. Apoptosis: an early event in metastatic inefficiency. Cancer Res 2001; 61: 333-338.

5. Martin SS, Vuori K. Regulation of Bcl-2 proteins during anoikis and amorphosis. Biochim Biophys Acta 2004; 1692: 145-157.

6. Eckhardt BL, Francis PA, Parker BS, Anderson RL. Strategies for the discovery and development of therapies for metastatic breast cancer. Nat Rev Drug Discov 2012; 11 : 479-497.

7. Newman DJ, Cragg GM. Natural products as sources of new drugs over the 30 years from 1981 to 2010. J Nat Prod 2012; 75: 311-335.

8. Newman DJ, Cragg GM. Microbial antitumor drugs: natural products of microbial origin as anticancer agents. Curr Opin Invest Drugs 2009; 10: 1280-1296.

9. Harvey AL. Natural products in drug discovery. Drug Discov Today 2008; 13: 894-901.

10. Li JW, Vederas JC. Drug discovery and natural products: end of an era or an endless frontier? Science 2009; 325: 161-165.

11. Danishefsky S. On the potential of natural products in the discovery of pharma leads: a case for reassessment. Nat Prod Rep 2010; 27: 1114-1116.

12. Wenzel SC, Muller R. Myxobacteria - 'microbial factories' for the production of bioactive secondary metabolites. Mol Biosyst 2009; 5: 567-574.

13. Altmann $\mathrm{KH}$, Memmert K. Epothilones as lead structures for new anticancer drugs pharmacology, fermentation, and structure-activity-relationships. Prog Drug Res 2008; 66; 273, 275-334.

14. Sandmann A, Sasse F, Muller R. Identification and analysis of the core biosynthetic machinery of tubulysin, a potent cytotoxin with potential anticancer activity. Chem Biol 2004; 11: 1071-1079.

15. Pando O, Dorner S, Preusentanz R, Denkert A, Porzel A, Richter W et al. First total synthesis of tubulysin B. Org Lett 2009; 11: 5567-5569.

16. Shibue T, Hirai T, Okamoto I, Morita N, Masu H, Azumaya I et al. Total syntheses of tubulysins. Chemistry 2010; 16: 11678-11688.

17. Ullrich A, Chai Y, Pistorius D, Elnakady YA, Herrmann JE, Weissman KJ et al. Pretubulysin, a potent and chemically accessible tubulysin precursor from Angiococcus disciformis. Angew Chem Int Ed Engl 2009; 48: 4422-4425.

18. Herrmann J, Elnakady YA, Wiedmann RM, Ullrich A, Rohde M, Kazmaier $U$ et al. Pretubulysin: from hypothetical biosynthetic intermediate to potential lead in tumor therapy. PLoS One 2012; 7: e37416.

19. Eirich J, Burkhart JL, Ullrich A, Rudolf GC, Vollmar A, Zahler S et al. Pretubulysin derived probes as novel tools for monitoring the microtubule network via activity-based protein profiling and fluorescence microscopy. Mol Biosyst 2012; 8: 2067-2075.

20. Rath S, Liebl J, Furst R, Ullrich A, Burkhart JL, Kazmaier U et al. Anti-angiogenic effects of the tubulysin precursor pretubulysin and of simplified pretubulysin derivatives. Br J Pharmacol 2012; 167: 1048-1061.

21. Kim SH, Ricci MS, El-Deiry WS. Mcl-1: a gateway to TRAIL sensitization. Cancer Res 2008; 68: 2062-2064.

22. Tao K, Fang M, Alroy J, Sahagian GG. Imagable $4 \mathrm{~T} 1$ model for the study of late stage breast cancer. BMC Cancer 2008; 8: 228

23. Cheng Y, Li G. Role of the ubiquitin ligase Fbw7 in cancer progression. Cancer Metast Rev 2012; 31: 75-87.

24. Inuzuka H, Fukushima $\mathrm{H}$, Shaik S, Liu P, Lau AW, Wei W. Mcl-1 ubiquitination and destruction. Oncotarget 2011; 2: 239-244.
25. Inuzuka H, Shaik S, Onoyama I, Gao D, Tseng A, Maser RS et al. SCF(FBW7) regulates cellular apoptosis by targeting MCL1 for ubiquitylation and destruction. Nature 2011; 471: 104-109.

26. Dumontet C, Jordan MA. Microtubule-binding agents: a dynamic field of cancer therapeutics. Nat Rev Drug Discov 2010; 9: 790-803

27. Kavallaris M. Microtubules and resistance to tubulin-binding agents. Nat Rev Cancer 2010; 10: 194-204

28. Jackson JR, Patrick DR, Dar MM, Huang PS. Targeted anti-mitotic therapies: can we improve on tubulin agents? Nat Rev Cancer 2007; 7: 107-117.

29. Sasse F, Steinmetz H, Heil J, Höfle G, Reichenbach H. Tubulysins, new cytostatic peptides from myxobacteria acting on microtubuli. Production, isolation, physico-chemical and biological properties. J Antibiot (Tokyo) 2000; 53: 879-885.

30. Khalil MW, Sasse F, Lünsdorf $H$, Elnakady YA, Reichenbach H. Mechanism of action of tubulysin, an antimitotic peptide from myxobacteria. Chembiochem 2006; 7: $678-683$

31. Steinmetz H, Glaser N, Herdtweck E, Sasse F, Reichenbach H, Höfle G. Isolation, crystal and solution structure determination, and biosynthesis of tubulysins-powerful inhibitors of tubulin polymerization from myxobacteria. Angew Chem Int Ed Engl 2004; 43: 4888-4892.

32. den Hollander MW, Gietema JA, de Jong S, Walenkamp AM, Reyners AK, Oldenhuis CN et al. Translating TRAIL-receptor targeting agents to the clinic. Cancer Lett 2013; 332: 194-201.

33. Sánchez-Pérez T, Ortiz-Ferrón G, López-Rivas A. Mitotic arrest and JNK-induced proteasomal degradation of FLIP and Mcl-1 are key events in the sensitization of breast tumor cells to TRAIL by antimicrotubule agents. Cell Death Differ 2010; 17: 883-894.

34. Wertz IE, Kusam S, Lam C, Okamoto T, Sandoval W, Anderson DJ et al. Sensitivity to antitubulin chemotherapeutics is regulated by MCL1 and FBW7. Nature 2011; 471: $110-114$

35. Wang TH, Wang HS, Ichijo H, Giannakakou P, Foster JS, Fojo T et al. Microtubuleinterfering agents activate c-Jun $\mathrm{N}$-terminal kinase/stress-activated protein kinase through both Ras and apoptosis signal-regulating kinase pathways. J Biol Chem 1998; 273: 4928-4936.

36. Stone AA, Chambers TC. Microtubule inhibitors elicit differential effects on MAP kinase (JNK, ERK, and p38) signaling pathways in human KB-3 carcinoma cells. Exp Cell Res 2000; 254: 110-119.

37. Nicoletti I, Migliorati G, Pagliacci MC, Grignani F, Riccardi C. A rapid and simple method for measuring thymocyte apoptosis by propidium iodide staining and flow cytometry. $J$ Immunol Methods 1991; 139: 271-279.

38. Pfaffl MW. A new mathematical model for relative quantification in real-time RT-PCR Nucleic Acids Res 2001; 29: e45.

39. Braig S, Kressirer CA, Liebl J, Bischoff F, Zahler S, Meijer L et al. Indirubin derivative 6BIO suppresses metastasis. Cancer Res 2013; 73: 6004-6012.

40. Häcker S, Dittrich A, Mohr A, Schweitzer T, Rutkowski S, Krauss J et al. Histone deacetylase inhibitors cooperate with IFN-gamma to restore caspase-8 expression and overcome TRAIL resistance in cancers with silencing of caspase-8. Oncogene 2009; 28 : $3097-3110$

cc)(1)(2) Cell Death and Disease is an open-access journal published by Nature Publishing Group. This work is licensed under a Creative Commons Attribution-NonCommercialShareAlike 3.0 Unported License. To view a copy of this license, visit http://creativecommons.org/licenses/by-nc-sa/3.0/ 